\title{
Herding: a new phenomenon affecting medical decision-making in multiple sclerosis care? Lessons learned from DIScUTIR MS
}

This article was published in the following Dove Press journal:

Patient Preference and Adherence

31 January 2017

Number of times this article has been viewed

\author{
Gustavo Saposnik ${ }^{1-3}$ \\ Jorge Maurino ${ }^{4}$ \\ Angel P Sempere ${ }^{5}$ \\ Christian C Ruff ${ }^{2}$ \\ Philippe N Tobler ${ }^{2}$ \\ 'Division of Neurology, Department \\ of Medicine, St Michael's Hospital, \\ University of Toronto, Toronto, \\ ON, Canada; ${ }^{2}$ Laboratory for Social \\ and Neural Systems Research, \\ Department of Economics, University \\ of Zurich, Zurich, Switzerland; \\ ${ }^{3} \mathrm{Li} \mathrm{Ka}$ Shing Knowledge Institute, \\ St Michael's Hospital, University \\ of Toronto, Toronto, ON, Canada; \\ ${ }^{4}$ Neuroscience Area, Medical \\ Department, Roche Farma, Madrid, \\ ${ }^{5}$ Department of Neurology, Hospital \\ General Universitario de Alicante, \\ Alicante, Spain
}

Purpose: Herding is a phenomenon by which individuals follow the behavior of others rather than deciding independently on the basis of their own private information. A herding-like phenomenon can occur in multiple sclerosis (MS) when a neurologist follows a therapeutic recommendation by a colleague even though it is not supported by best practice clinical guidelines. Limited information is currently available on the role of herding in medical care. The objective of this study was to determine the prevalence (and its associated factors) of herding in the management of MS.

Methods: We conducted a study among neurologists with expertise in MS care throughout Spain. Participants answered questions regarding the management of 20 case scenarios commonly encountered in clinical practice and completed 3 surveys and 4 experimental paradigms based on behavioral economics. The herding experiment consisted of a case scenario of a 40 -year-old woman who has been stable for 3 years on subcutaneous interferon and developed a self-limited neurological event. There were no new magnetic resonance imaging (MRI) lesions. Her neurological examination and disability scores were unchanged. She was advised by an MS neurologist to switch from interferon to fingolimod against best practice guidelines. Multivariable logistic regression analysis was conducted to evaluate factors associated with herding.

Results: Out of 161 neurologists who were invited to participate, 96 completed the study (response rate: $60 \%$ ). Herding was present in 75 (78.1\%), having a similar prevalence in MS experts and general neurologists $(68.8 \%$ vs $82.8 \% ; P=0.12)$. In multivariate analyses, the number of MS patients seen per week was positively associated with herding (odds ratio [OR] 1.08, 95\% CI 1.01-1.14). Conversely, physician's age, gender, years of practice, setting of practice, or risk preferences were not associated with herding.

Conclusion: Herding was a common phenomenon affecting nearly 8 out of 10 neurologists caring for MS patients. Herding may affect medical decisions and lead to poorer outcomes in the management of MS.

Keywords: multiple sclerosis, herding, disease-modifying therapy, neuroeconomics, decisionmaking, risk aversion

\section{Introduction}

Medical decisions are difficult, especially when considering that they affect others: our patients and those who are close to them. ${ }^{1}$ The social aspects of medical decisions can extend beyond patients and include also colleagues, potentially with different expertise. For example, physicians may follow diagnostic and therapeutic recommendations given by other colleagues even if that decision is wrong or not supported by best practice guidelines in the field. Despite many years of medical training, physicians
Correspondence: Gustavo Saposnik Division of Neurology, Departmen of Medicine, St Michael's Hospital, University of Toronto, 55 Queen Street East, Toronto, ON M5C IR6, Canada $\mathrm{Tel}+\mathrm{I} 4168645155$

Fax + 4168645150 Email saposnikg@smh.ca 
have limited education in decision-making and the social factors that influence it. ${ }^{2}$ However, it is unclear to what degree physicians succumb to social influence and make erroneous decisions as a consequence.

In behavioral economics, herding is a phenomenon by which individuals follow others or imitate group behaviors rather than deciding independently on the basis of their own private information. The concept of herding has its roots in the work initiated by Keynes, ${ }^{3}$ who focused on the motivations to imitate and follow the crowd under uncertainty. Keynes ${ }^{3}$ conceived herding as a response to uncertainty when individuals perceive their own ignorance on a specific topic. ${ }^{3}$ As a result, individuals may follow others' recommendations because they believe that the rest of the crowd (or someone admired or well respected) is better informed. The propagation of information from a mate or the crowd rather than relying on one's own private signal can lead to a "cascade" of erroneous information. This phenomenon is one of the explanations brought forward for irrational financial speculation (eg, financial "bubbles"), which repeatedly affects economies. ${ }^{4}$

Multiple sclerosis (MS) is a chronic inflammatory demyelinating condition that affects the central nervous system. Due to its progressive nature leading to neurological disability, MS has a strong physical and emotional impact on patients and those who are close to them. The earlier use of high-efficacy disease-modifying therapies (DMTs) is recommended to slow the course of MS when there is evidence of clinical and radiological progression..$^{5-7}$ Failure to adhere to this recommendation may result in incorrect patient and family expectations and potentially suboptimal advice, treatment, and outcomes.

Herding-like behavior when following erroneous decisions may facilitate failure to implement best clinical practice (also called "negative herding"). For example, residents routinely follow staff recommendations, general practitioners follow specialist's advice, and specialists commonly follow experts in the field - also called "opinion leaders" even though they may provide erroneous recommendations. Although the available information from guidelines is public, medical decisions are based on private information. Each physician performs an individual assessment weighing patients' factors differently (not shared with others) before making a decision. As a result of this herding-like behavior, a sequence of suboptimal or erroneous decisions may arise, which could lead to medical errors as observed in clinical practice and legal medicine. ${ }^{8}$

We tested the hypothesis that neurologists caring for MS patients are prone to herding-like behavior when following erroneous recommendations provided by colleagues. In this study, we assessed the prevalence of herding-like behavior (and associated contributing factors) in typical clinical decisions among physicians caring for MS patients. We specifically selected this medical condition given that MS care involves complex medical decisions and requires consideration of multiple short- and long-term factors (eg, neuroimaging results, disease progression, patient's characteristics, and their preferences) and disease-modifying agents.

\section{Methods}

We conducted a web-based study (DIScUTIR MS) using the Qualtrics platform. It comprised $20 \mathrm{MS}$ case vignettes regarding therapeutic recommendations among practicing neurologists from Spain from November 3, 2015 to March 31, 2016. The study was approved by the Research Ethics Board of St Michael's Hospital, University of Toronto, Canada. Written informed consent was obtained from all participants. We also evaluated neurologists' ambiguity aversion, risk aversion, and tolerance to uncertainty. Further details of the protocol were published elsewhere. ${ }^{9}$

Recent meta-analysis confirmed that fingolimod, natalizumab, and alemtuzumab are the best available choices for preventing clinical relapses in patients with relapsingremitting multiple sclerosis (RRMS). ${ }^{10}$ The current landscape of DMTs for the treatment of RRMS includes first-line therapies (beta interferons, glatiramer acetate, teriflunomide, and dimethyl fumarate) and second-line therapies (fingolimod, natalizumab, and alemtuzumab). National and regional recommendations include escalating therapy from a first-line agent to a second-line agent when there is evidence of clinical and radiological disease progression. ${ }^{6,11-15}$ However, switching to a second-line agent is not supported by best practice recommendations if an MS patient has been clinically stable, with no new lesions on a follow-up magnetic resonance imaging (MRI) or no progression on the disability scale. ${ }^{6,11-15}$ For the current analysis, we used the aforementioned scheme according to the current clinical practice. MS case scenarios followed best practice guidelines supervised by experts in the field.

\section{Participants}

Practicing neurologists actively involved in the care of MS patients from across Spain were invited to participate in our study by the Spanish Society of Neurology (Sociedad Española de Neurologia-SEN). Physicians who primarily cared for MS patients were classified as "MS specialists". All the remaining physicians were classified as "general neurologists". All participants received compensation for completing the survey. 


\section{Herding experiment}

Participants were exposed to a case scenario (illustrated in the following sections) describing a woman who has been stable on beta-interferon for 3 years with no evidence of radiological or disease progression (stable Expanded Disability Status Scale [EDSS] score of 1.5) and was assessed by an MS neurologist who recommended escalating therapy to fingolimod when not supported by the currently available recommendations. ${ }^{11,12,16}$ Herding-like behavior arose when participants followed an erroneous recommendation (negative herding) from an MS colleague instead of making an individual evidence-based decision.

Participants read the following case scenario:

A 40-year-old woman was diagnosed with MS 3 years ago.

She has been taking subcutaneous interferon beta-1a with no significant side effects. Three months ago, she developed bilateral leg weakness and urinary urgency, which resolved within 2-3 weeks. An MRI of the brain at that time revealed a total of 10 periventricular and juxtacortical T2 lesions, similar to her baseline MRI. There were no gadoliniumenhanced T1 lesions. Her neurological examination, as well as her EDSS score of 1.5 is unchanged from last year? She expressed some concerns about her recent symptoms while being on DMTs. She was seen by an MS colleague in your absence who recommended switching to fingolimod. She came back to your office to get your opinion.

Next, participants were asked what treatment they would recommend, including 1) support starting her on fingolimod as recommended by your MS colleague or 2) continue on subcutaneous interferon beta-1a.

\section{Outcome measures}

The primary outcome of the study was the proportion of participants who exhibited herding following a colleague recommendation when not supported by the current available guidelines. ${ }^{11,12,16}$ Secondary outcomes included the association of herding with demographic information and physicians' characteristics.

\section{Statistical analysis}

Chi-square tests were used to compare categorical variables; appropriate parametric or nonparametric tests were used to compare mean and median differences for continuous or ordinal data. A multivariable logistic regression analysis with adjustment for age, years of experience, number of MS patients seen per week, coauthor of a peer-reviewed journal in the last 3 years, and practice setting (academic vs community institution) was completed to evaluate the factors associated with herding. A sensitivity analysis was completed by adding neurologist's risk aversion, ambiguity aversion, and low tolerance to uncertainty. All the tests were 2-tailed, and the alpha level was set to $P<0.05$.

\section{Results}

Overall, 161 neurologists were invited to participate in the study; 136 cooperated (cooperation rate $84.5 \%$ ) and 96 completed the survey (response rate 60\%). There was representation from all regional territories except the Canary Islands. Baseline characteristics of responders are summarized in Table 1. The mean (SD) age was $39.5( \pm 8.5)$ years; $51(53 \%)$ were female. The median time for completing the study was 39 minutes (interquartile range [IQR] 30-52 minutes). We found no difference in demographic characteristics between participants and nonparticipants.

Herding was observed in 75 (78.1\%) participants, having a similar prevalence in MS experts and general neurologists (68.8\% vs $82.8 \% ; P=0.12$; Figure 1A). Participants who exhibited herding-like behavior had larger volume of MS patients seen per week than the non-herding group ( 22 vs 12 patients, respectively; $P=0.03$ ). There was no difference in herding by practice setting (physicians practicing in nonacademic vs academic institutions: $85.2 \%$ vs $75.4 \%$, $P=0.30)$ or years of experience $(P=0.58)$. There was also no

Table I Baseline characteristics of participants

\begin{tabular}{ll}
\hline Characteristics & No of participants (\%) \\
\hline Age (mean \pm SD), in years & $39.5 \pm 8.5$ \\
Gender & \\
$\quad$ Female & $51(53.1)$ \\
Specialty & $64(66.7)$ \\
$\quad$ MS specialist & \\
$\quad$ primarily sees MS patients) & $32(33.3)$ \\
$\quad$ General neurologist who cares & \\
for MS patients & \\
Practice setting & $48(50.0)$ \\
Academic & $26(27.1)$ \\
Community & $21(21.9)$ \\
Both (academic and community) & I (I.0) \\
Other & \\
$\%$ time in clinical practice & $70(72.9)$ \\
$\quad>75 \%$ & $14.1 \pm 10$ \\
Years in practice, mean ( \pm SD) & $20 \pm 15$ \\
MS patients seen per week, mean $( \pm S D)$ & $56(58)$ \\
Attended latest ECTRIMS conference & $79(82.3)$ \\
Author of a peer-reviewed publication & \\
in the last 3 years & \\
Personality characteristics & $19(20.0)$ \\
Risk aversion & $22(22.9)$ \\
Ambiguity aversion & $4 I(42.7)$ \\
Low tolerance to uncertainty &
\end{tabular}

Abbreviations: ECTRIMS, European Committee for Treatment and Research of Multiple Sclerosis; MS, multiple sclerosis. 

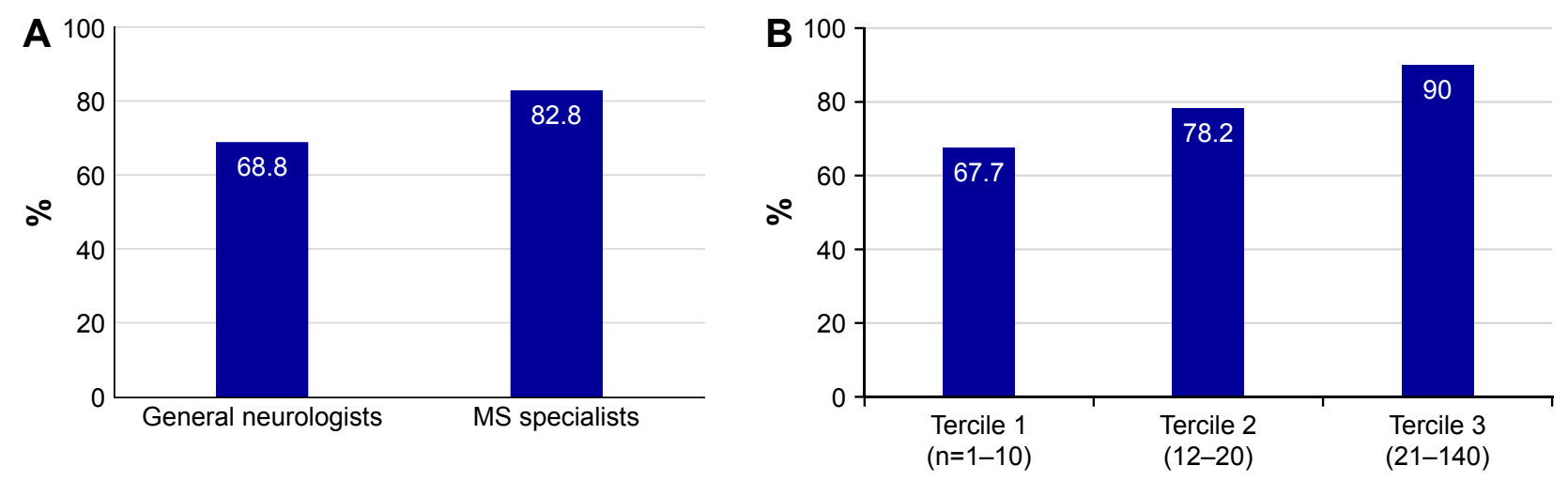

Figure I Prevalence of herding-like behavior according to specialty and volume of MS patients.

Notes: (A) Herding-like behavior in MS specialists and general neurologists. (B) Prevalence of herding-like behavior by volume of MS patients seen per week (in terciles). Abbreviation: MS, multiple sclerosis.

difference in time taken for completing the herding experiment between groups (herding $=95$ vs 102 seconds for those who did not herd; $P=0.76$ ) or in the prevalence of herding among participants completing the study below and above the 90th percentile (60 minutes; $82.8 \%$ vs $71.1 \% ; P=0.18$ ).

In multivariate analysis after adjusting for confounders, the number of MS patients seen per week was associated with herding (odds ratio [OR] 1.08, 95\% CI 1.01-1.16; Table 2 and Figure 1B). There was no association between herding and demographic factors or participants' characteristics. There was an adequate calibration (goodness-of-fit test 0.77 ) and discrimination (area under the curve 0.808 ) of the model. The results remained consistent after adjusting for neurologists' personality traits (risk aversion, aversion to ambiguity, and low tolerance to uncertainty) and time taken for completing the experiment (data not shown).

\section{Discussion}

MS patients and their treating physicians are routinely confronted with uncertainties concerning diagnosis, prognosis, disease course, and DMTs. ${ }^{17}$ In this study, we evaluated whether neurologists and MS experts follow the erroneous

Table 2 Factors associated with herding-like behavior

\begin{tabular}{ll}
\hline Outcomes & $\begin{array}{l}\text { Adjusted model for } \\
\text { herding*; OR (95\% CI) }\end{array}$ \\
\hline Age, in years & $1.02(0.94-1.11)$ \\
Gender, male & $0.58(0.19-1.76)$ \\
Time in practice, per year & $0.95(0.88-1.02)$ \\
Coauthor of a recent peer-reviewed article & $0.36(0.10-1.23)$ \\
Practice setting (academic vs community) & $0.29(0.07-1.16)$ \\
Low tolerance to uncertainty & $0.41(0.13-1.26)$ \\
Number of MS patients seen per week & $1.08(1.01-1.15)$ \\
\hline
\end{tabular}

Notes: *Model derived from logistic regression including all variables of interest (age, gender, number of MS patients seen per week, practice setting, academic profile, and tolerance to uncertainty).

Abbreviations: MS, multiple sclerosis; OR, odds ratio. advice provided by another colleague - a herding-like behavior. We found that nearly 8 out of 10 participants followed the recommendation of an MS colleague when not supported by local and international best practice guidelines. ${ }^{11,12,16}$ In the multivariable analysis, a higher volume of MS patients (identified by the estimated number of MS patients seen per week) were associated with herding-like behavior. Traditional demographic factors, medical experience, and practice setting were not related to herding-like behavior. This phenomenon may be explained by mental fatigue caused by high volume of consultations in medical situations with cognitively demanding decisions. ${ }^{18,19}$ For example, a study of 585 malpractice errors found that the main contributing factor was "role overload" in more than one-third of cases. ${ }^{20}$

Another potential explanation is derived from Keynes' quotation: "Worldly wisdom teaches that it is better for reputation to fail conventionally than to succeed unconventionally". As such, participants may have chosen switching therapy with the intention of being conventional (as recommended by an MS colleague).

Herding-like behavior is a novel concept in the medical field. To the best of our knowledge, there were no studies evaluating herding-like behavior in MS or other medical conditions. The underlying concept of why people may mistakenly follow others' decisions was introduced by Keynes. ${ }^{3} \mathrm{He}$ believed that people purchase different objects (from a tulip bulb to a car or a house) at a seemingly exorbitant price not because they independently believe that the object is worth the cost, but because they believe that other people think that it is. In other words, some individuals believe that others (either peers or experts) may have better information than their own. ${ }^{21}$ This particular situation may more commonly occur under uncertainty. In repetitive situations, followers are being followed by other individuals, leading to further dissemination of misinformation. 
Given the importance of real-world evidence providing information on the long-term effectiveness and safety of disease-modifying agents, the incorporation of herding is likely to affect therapeutic decisions in MS care. ${ }^{22}$

Outside of the medical field, herding-like behavior has been described too. In aeronautics, the disintegration of the Space Shuttle Columbia in $2003^{23}$ and the crash of Korean Air flight 801 in $1997^{24}$ are examples of how decision-makers mistakenly followed the recommendation of other team members contributing to these catastrophes.

The presence of herding-like behavior in the medical domain has practical clinical and legal implications. For example, worse outcomes (more clinical relapses, disease progression) could be expected if a second physician follows the mistaken advice given by an MS colleague. Similarly, the initial erroneous interpretation of a test result (eg, a computed tomography scan of the head in a young individual with new symptoms and a focal neurological deficit) or the lack of initiation of an appropriate treatment (eg, prescription of an antibiotic for a patient presenting symptoms suggestive of a meningitis) followed by the second physician who started the shift may lead to poor clinical outcomes. Some medical environments may be riskier than others. For example, emergency care in high-volume facilities with high turnover of health care personnel (eg, shorter shifts) under time constraints may predispose to fragmented care, suboptimal communication, or discussion of the clinical plan, which could lead to herding-like behavior. Specifically, newly starting physicians may not be properly informed about the current status of patients in the ward and have limited time to initiate a new medical history, physical examination, and reassessment of test results ordered by the previous colleague. ${ }^{25,26}$

\section{Limitations}

Our study has some limitations that deserve comment. First, the case scenario may not fully capture actual decisions made in clinical practice. Second, our findings should be viewed as exploratory given the relatively small sample size. Finally, our study was conducted in Spain exclusively, thus limiting the generalizability of our results to other cultural contexts.

Despite these limitations, our study constitutes the first step toward understanding the role of herding in medical decisions. Furthermore, our findings highlight the influence of herding on therapeutic decisions in MS patients beyond demographic factors, medical expertise, practice setting, and patients' factors or their treatment preferences. Using a novel approach that combines case vignettes with the elicitation of herding-like behavior through experiments from behavioral economics, we were able to expand our current understanding of decision-making in MS care.

Our results may not only be relevant for MS care but also be seen as the initial action to increase awareness for transferred misinformation among physicians. Herding-like behavior could represent the root of medicolegal cases as it may trigger a "string of mistakes" or cascade of errors due to many constraints in medical care (eg, limited allocated time, high volume of consultations, fragmented care, suboptimal communication, or discussions between the starting and exiting physician working shifts). ${ }^{8}$

\section{Conclusion}

Our study shows that nearly 8 out of 10 neurologists may exhibit negative herding by following an erroneous recommendation provided by an MS colleague. High patient volume was the single factor associated with herding. Further research is needed to determine the prevalence of herding (and its associated factors) in other medical conditions that could lead to poorer patients' outcomes and result in medico-legal complaints. ${ }^{27}$

\section{Acknowledgments}

The authors are most grateful to all physicians participating in the DIScUTIR MS study. We appreciate the support from the Department of Economics at the University of Zurich by facilitating access to Qualtrics platform.

\section{Disclosure}

The study was sponsored by the Sociedad Española de Neurologia (SEN) and funded by an operating grant from Roche Farma Spain. The sponsors were not involved in the design, execution, analysis, and interpretation or reporting of the results. Dr Gustavo Saposnik is supported by the Distinguished Clinicians Scientist Award from HSFC. Dr Jorge Maurino is an employee of Roche Farma Spain. Prof Philippe Tobler and Christian Ruff were funded by the Swiss National Science Foundation (PNT: PP00P1_150739, CRSII3_141965, and 00014_165884,CCR:105314_152891, CRSII3_141965, and 320030_143443). The authors report no other conflicts of interest in this work.

\section{References}

1. Glimcher P, Fehr E. Neuroeconomics: Decision Making and the Brain. 2nd ed. San Diego, CA: Academic Press; 2014.

2. Dijkstra IS, Pols J, Remmelts P, Brand PL. Preparedness for practice: a systematic cross-specialty evaluation of the alignment between postgraduate medical education and independent practice. Med Teach. 2015; 37(2):153-161.

3. Keynes JM. A Treatise on Money. London: Macmillan; 1930. 
4. Baddeley M. Herding, social influence and economic decision-making: socio-psychological and neuroscientific analyses. Philos Trans $R$ Soc Lond B Biol Sci. 2010;365(1538):281-290.

5. Noyes K, Weinstock-Guttman B. Impact of diagnosis and early treatment on the course of multiple sclerosis. Am J Manag Care. 2013; 19(17 suppl):s321-s331.

6. Sormani MP, Rio J, Tintore M, et al. Scoring treatment response in patients with relapsing multiple sclerosis. Mult Scler. 2013;19(5):605-612.

7. Duquette P, Giacomini PS, Bhan V, Hohol M, Schecter R. Balancing early aggression against risk of progression in multiple sclerosis. Can J Neurol Sci. 2016;43(1):33-43.

8. Woolf SH, Kuzel AJ, Dovey SM, Phillips RL Jr. A string of mistakes: the importance of cascade analysis in describing, counting, and preventing medical errors. Ann Fam Med. 2004;2(4):317-326.

9. Saposnik G, Sempere AP, Raptis R, Prefasi D, Selchen D, Maurino J. Decision making under uncertainty, therapeutic inertia, and physicians' risk preferences in the management of multiple sclerosis (DIScUTIR MS). BMC Neurol. 2016;16(1):58.

10. Tramacere I, Del Giovane C, Salanti G, D’Amico R, Filippini G. Immunomodulators and immunosuppressants for relapsing-remitting multiple sclerosis: a network meta-analysis. Cochrane Database Syst Rev. 2015;2015(9):CD011381.

11. Freedman MS, Selchen D, Arnold DL, et al; Canadian Multiple Sclerosis Working Group. Treatment optimization in MS: Canadian MS Working Group updated recommendations. Can J Neurol Sci. 2013;40(3): 307-323.

12. Wattjes MP, Rovira A, Miller D, et al; MAGNIMS Study Group. Evidence-based guidelines: MAGNIMS consensus guidelines on the use of MRI in multiple sclerosis - establishing disease prognosis and monitoring patients. Nat Rev Neurol. 2015;11(10):597-606.

13. Prosperini L, Mancinelli CR, De Giglio L, De Angelis F, Barletta V, Pozzilli C. Interferon beta failure predicted by EMA criteria or isolated MRI activity in multiple sclerosis. Mult Scler. 2014;20(5):566-576.

14. Correale J, Abad P, Alvarenga R, et al. Management of relapsing-remitting multiple sclerosis in Latin America: practical recommendations for treatment optimization. J Neurol Sci. 2014;339(1-2):196-206.

15. García Merino A, Ramón Ara Callizo J, Fernández Fernández O, Landete Pascual L, Moral Torres E, Rodríguez-Antigüedad Zarrantz A. Consensus statement on the treatment of multiple sclerosis by the Spanish Society of Neurology in 2016. Neurologia. Epub 2016 May 5.
16. Sormani MP, Gasperini C, Romeo M, et al. Assessing response to interferon-beta in a multicenter dataset of patients with MS. Neurology. 2016;87(2):134-140.

17. Bermel RA, You X, Foulds P, et al. Predictors of long-term outcome in multiple sclerosis patients treated with interferon beta. Ann Neurol. 2013;73(1):95-103.

18. Linder JA, Doctor JN, Friedberg MW, et al. Time of day and the decision to prescribe antibiotics. JAMA Intern Med. 2014;174(12):2029-2031.

19. Danziger S, Levav J, Avnaim-Pesso L. Extraneous factors in judicial decisions. Proc Natl Acad Sci U S A. 2011;108(17):6889-6892.

20. Björkstén KS, Bergqvist M, Andersén-Karlsson E, Benson L, Ulfvarson J. Medication errors as malpractice-a qualitative content analysis of 585 medication errors by nurses in Sweden. BMC Health Serv Res. 2016;16(1):431

21. Muchnik L, Aral S, Taylor SJ. Social influence bias: a randomized experiment. Science. 2013;341(6146):647-651.

22. Prosperini L, Sacca F, Cordioli C, et al. Real-world effectiveness of natalizumab and fingolimod compared with self-injectable drugs in non-responders and in treatment-naïve patients with multiple sclerosis. J Neurol. Epub 2016 Nov 22.

23. Columbia Accident Investigation Board [homepage on the Internet]. Washington, DC: National Aeronautics and Space Administration and the Government Printing Office; 2011. Available from: https:// spaceflight.nasa.gov/shuttle/archives/sts-107/investigation/CAIB_ medres_full.pdf. Accessed January 17, 2017.

24. Bordallo attends unveiling of painting depicting 1997 Korean Air Crash [webpage on the Internet]. Guam: Official Guam Crash Site Center Korean Air Fit 801. Available from: http://ns.gov.gu/guam/indexmain. html. Accessed January 17, 2017.

25. Woiceshyn J, Blades K, Pendharkar SR. Integrated versus fragmented implementation of complex innovations in acute health care. Health Care Manage Rev. 2017;42(1):76-86.

26. Wohlauer M. Fragmented care in the era of limited work hours: a plea for an explicit handover curriculum. BMJ Qual Saf. 2012;21(suppl 1): i16-i18.

27. Emanuel EJ, Ubel PA, Kessler JB, et al. Using behavioral economics to design physician incentives that deliver high-value care. Ann Intern Med. 2016;164(2):114-119.
Patient Preference and Adherence

\section{Publish your work in this journal}

Patient Preference and Adherence is an international, peer-reviewed, open access journal that focuses on the growing importance of patient preference and adherence throughout the therapeutic continuum. Patient satisfaction, acceptability, quality of life, compliance, persistence and their role in developing new therapeutic modalities and compounds to optimize

\section{Dovepress}

clinical outcomes for existing disease states are major areas of interest for the journal. This journal has been accepted for indexing on PubMed Central. The manuscript management system is completely online and includes a very quick and fair peer-review system, which is all easy to use. Visit http://www. dovepress.com/testimonials.php to read real quotes from published authors. 